Supporting Information for Publication

\title{
Evolution of the Magnetic and Structural Properties with the Chemical Composition in Oleate-capped $\mathrm{Mn}_{x} \mathrm{Co}_{1-x} \mathrm{Fe}_{2} \mathrm{O}_{4}$ Nanoparticles
}

\author{
Marco Sanna Angotzi, ${ }^{1,2}$ Valentina Mameli,,${ }^{1,2 *}$ Dominika Zákutná, ${ }^{3}$ Denisa Kubániová, ${ }^{4}$ Claudio \\ Cara, ${ }^{1,2}$ Carla Cannas ${ }^{1,2}$ \\ ${ }^{1}$ University of Cagliari, Department of Chemical and Geological Sciences, Cittadella Universitaria S.P. Monserrato Sestu \\ Km 0.700, 09042 Monserrato, Italy. \\ ${ }^{2}$ Consorzio Interuniversitario Nazionale per La Scienza e Tecnologia Dei Materiali (INSTM), Cagliari Unit, Via Giuseppe \\ Giusti 9, Firenze, 50121, Italy \\ ${ }^{3}$ Department of Inorganic Chemistry, Charles University, Hlavova 2030, 128 40, Prague 2, Czech Republic \\ ${ }^{4}$ Department of Low Temperature Physics, Faculty of Mathematics and Physics, Charles University, V Holešovičkách 2, \\ Prague, 180 00, Czech Republic \\ *Corresponding author: valentina.mameli@unica.it
}




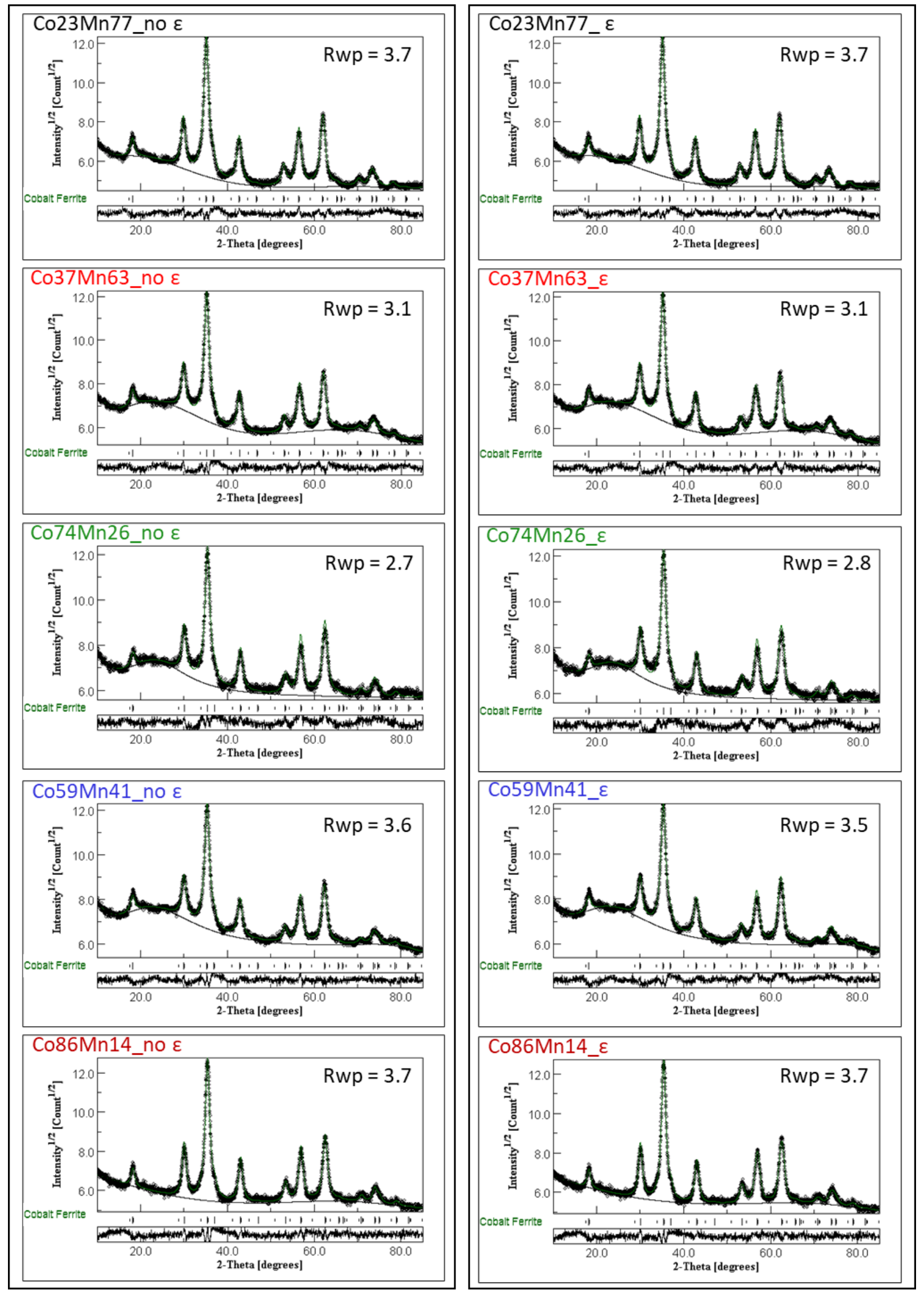

Figure S1 Rietveld refinement on the XRD patterns without (left) and with (right) microstrain. 


\section{S2. TEM analysis}
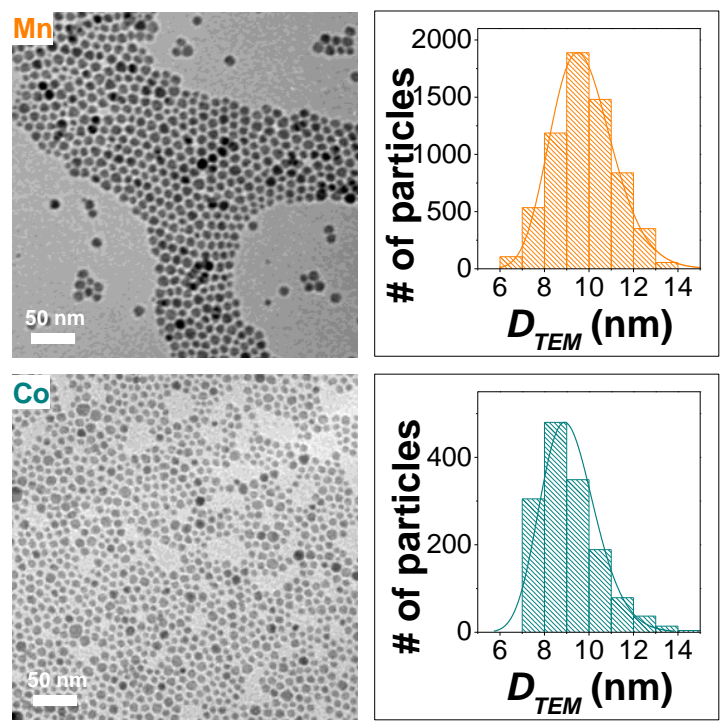

Figure S2 TEM bright-field micrographs and particle size distributions (as number (\#) of particles per class of TEMderived diameter, $\mathrm{D}_{\text {TEM }}$ ) of the $\mathrm{MnFe}_{2} \mathrm{O}_{4}$ and $\mathrm{CoFe}_{2} \mathrm{O}_{4}$ nanoparticles.

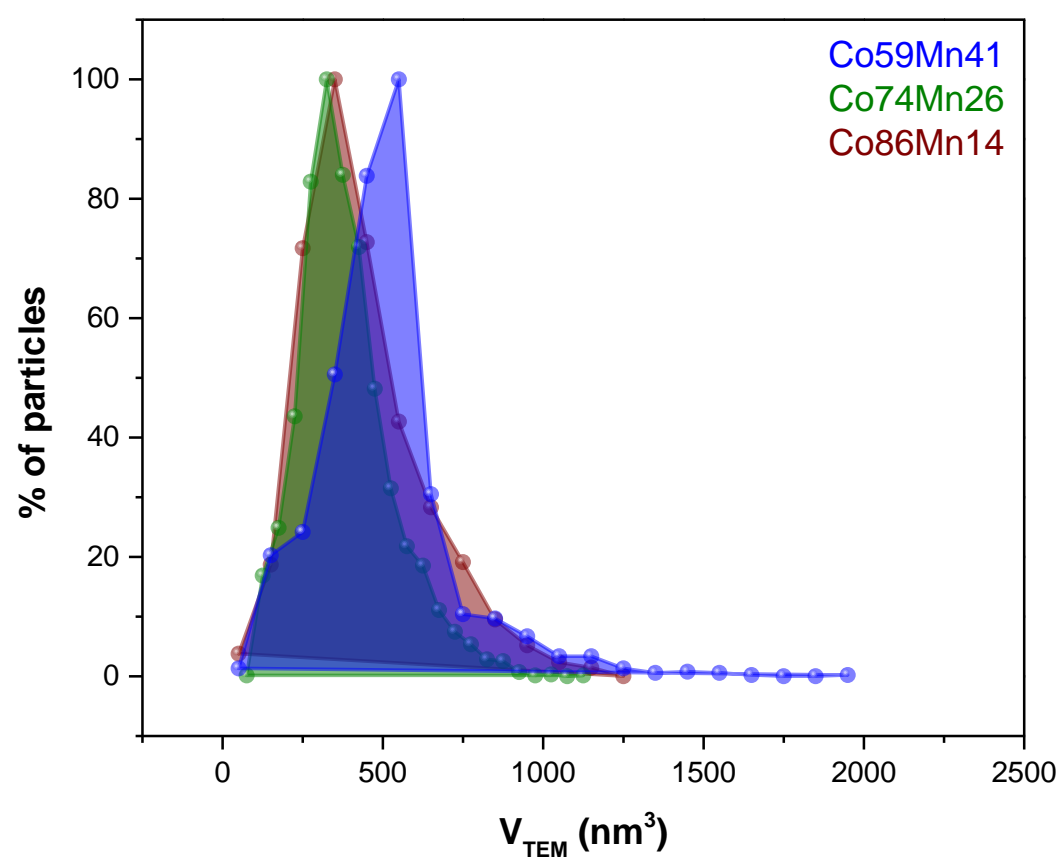

Figure S3 TEM particle volume distributions (as percentage (\%) of particles per class of TEM-derived volume, $\mathrm{V}_{\text {TEM }}$ ) of the Co59Mn41 (blue), Co74Mn26 (olive), and Co86Mn14 (wine) samples. 


\section{S3. DC magnetometry}
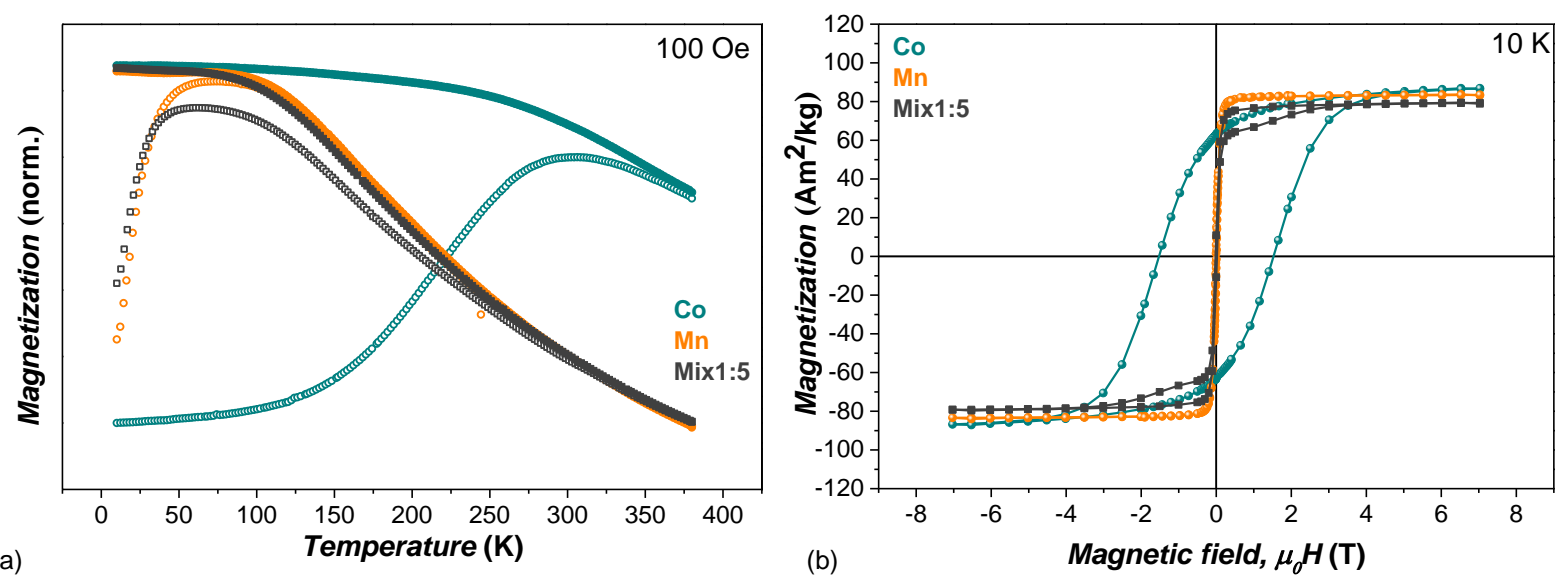

Figure S4 ZFC (empty circles) and FC (full circles) curves recorded at low external magnetic field (10 mT) (a) and magnetisation isotherms recorded at $10 \mathrm{~K}$ (b) of the $\mathrm{MnFe}_{2} \mathrm{O}_{4}, \mathrm{CoFe}_{2} \mathrm{O}_{4}$ nanoparticles, and their physical mixture (weight ratio equal to $1: 5)$. 


\section{S4. Magnetic domain size and moment distribution by numerical inversion}

Magnetic moment and domain size distributions were obtained by a non-regularized inversion method by means of MINORIM software, ${ }^{1}$ and a regularized inversion method. ${ }^{2,3}$ The input data were pre-treated by mathematical interpolation in the case of MINORIM method in order to get a 1000 points-curve. The magnetic domain size distributions from which the mean magnetic moments were extracted (Table 2 in the main manuscript) were derived from the saturation magnetization $\left(\mathrm{M}_{\mathrm{s}}\right)$ weighted dipole moment distribution of powder particles from MINORIM ${ }^{1}$ obtained by using the conditions reported in the experimental section of the main manuscript (i.e., a cut off of $1500000 \mathrm{~A} / \mathrm{m}$ for correcting the diamagnetic contribution, minimum and maximum dipole moments equal to $1 \cdot 10^{-}$ 22 and $1 \cdot 10^{-17} \mathrm{~A} \cdot \mathrm{m}^{2}$, respectively, and 36 bins for 6 subsets, Langevin dipole domain mode). These conditions led to monomodal distributions, as shown in Figure S5 and Figure S6.

MINORIM is a model-independent, non-regularized inversion method that does not assume monomodality nor other prior knowledge of the shape of the particle size or magnetic dipole moments' distributions, and it uses a non-negative least-squares procedure (NNLS), in order to avoid the obtainment of negative unphysical results. ${ }^{1}$ From this method, it is possible to obtain both number-weighted and $\mathrm{M}_{\mathrm{s}}$-weighted distributions (Figure S5, Figure S6).

Figure S7 shows the comparison between the number-weighted magnetic domain diameter distributions with the particle diameter distributions obtained from TEM. As can be seen, the magnetic distribution is always shifted towards lower size values if compared to the TEM one, with a difference in the maxima of about 6-7 $\mathrm{nm}$. This large discrepancy is much higher than that between

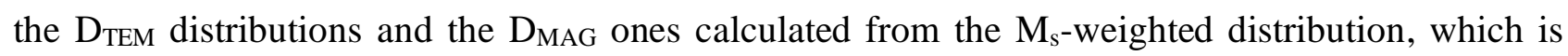
about 2-4 nm. First of all, we should consider that the NNLS of MINORIM has been developed for colloidal dispersion of non-interacting superparamagnetic nanoparticles, while the samples were analysed as powders, and they probably feature dipolar interactions. Nevertheless, the observed discrepancy could be an indication of the presence of magnetic spin disorder phenomena since the difference is higher than 1-2 $\mathrm{nm}$ in terms of diameter. ${ }^{1,2}$

However, since the size distribution obtained by MINORIM strongly depends on the chosen values for the number of bins and subsets and minimum and maximum values of dipole moment (Figure S5, Figure S6), polymodal distributions can also be obtained for the Co-Mn mixed ferrite samples.

In particular, if the method implemented in MINORIM is left to optimize the total bins and subsets, polymodal distributions with well-separated populations are obtained for all samples instead of the monomodal ones observed with 36 bins and 6 subsets. Nevertheless, it is worth noting that the broad monomodal distributions comprise all those single contributions for all samples (Figure S5).

However, fruitful information can be obtained when comparing the number-weighted curves obtained for optimized number of bins and subsets for the different samples (Figure S6). Indeed, the Mn-richest samples appear more similar with two main contributions below $5 \mathrm{~nm}$, while the Corichest ones feature one main population. In addition to these main populations, all samples are also characterised by a not negligible ensemble of magnetic domains between 7 and $8 \mathrm{~nm}$. The sample Co59Mn41 has an opposite trend in the intensity of the two main populations with respect to those of the Mn-richest samples (Co23Mn77, Co37Mn63), in agreement with the TEM data (Figure 2, Figure S3) and the ZFC curves and energy barrier distribution (Figure 3).

In addition, based on the references ${ }^{2,3}$ the magnetic moment distributions for all samples were also obtained by a regulized numerical inversion again with no ad hoc assumptions regarding the line 
shape of the extracted distribution functions and based on a NNLS procedure. The results are shown in Figure S8 and reveal once again polymodal distribution with two main populations, whose intensities are similar for all samples but Co59Mn41 and Co74Mn26. In particular, for these two samples the intensity of the two populations is opposite, in agreement with the behaviour observed by the MINORIM, TEM and DC magnetometry data.
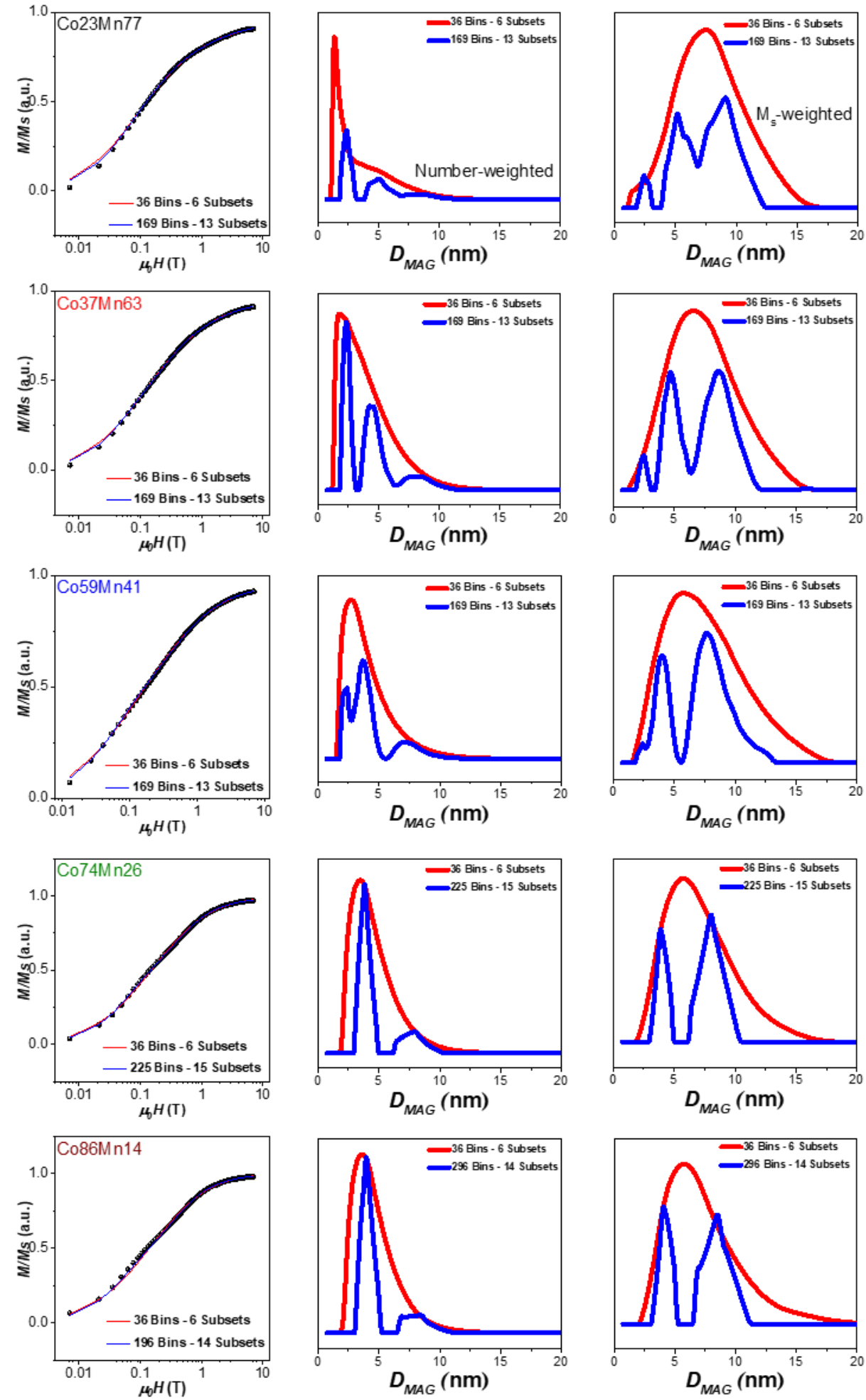

Figure S5 Field-dependent magnetization refinement of the $300 \mathrm{~K}$ magnetic field dependence of the magnetization for the Co-Mn mixed ferrite samples by using MINORIM ${ }^{1}$ (left), and resulting number-weighted (centre) and $\mathrm{M}_{\mathrm{s}}$-weighted (right) magnetic domain size distributions obstained for different selections of number of bins and subsets. 

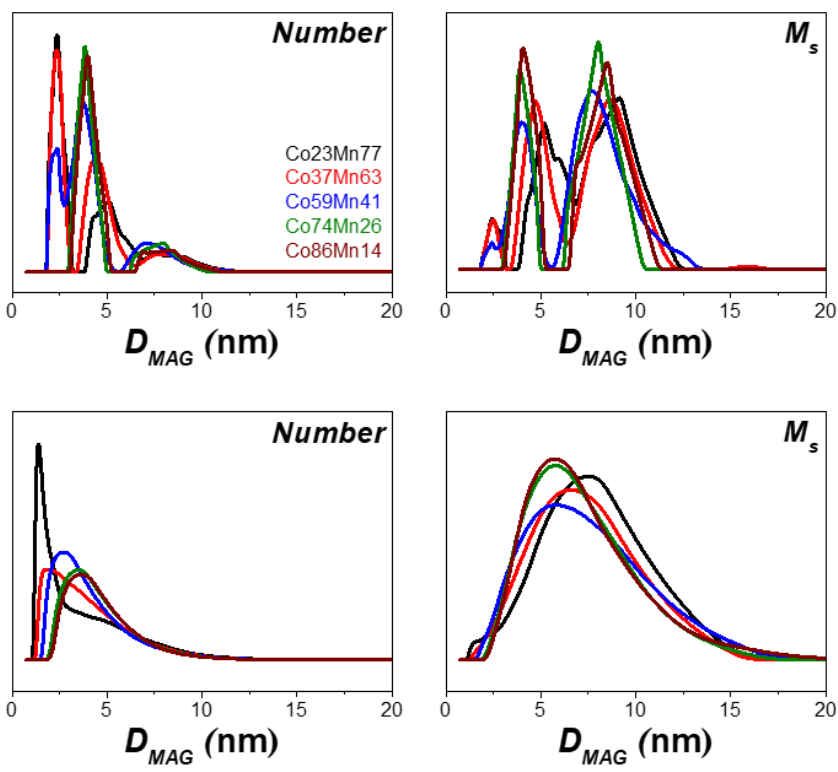

Figure S6 Comparison of number-weighted (left) and $\mathrm{M}_{\mathrm{s}}$-weighted (right) magnetic domain size distributions for the CoMn mixed ferrite samples obstained by using MINORIM ${ }^{1}$ for optimesed bins and subsets (upper graphs) and 36 bins and 6 subsets (bottom graphs). 

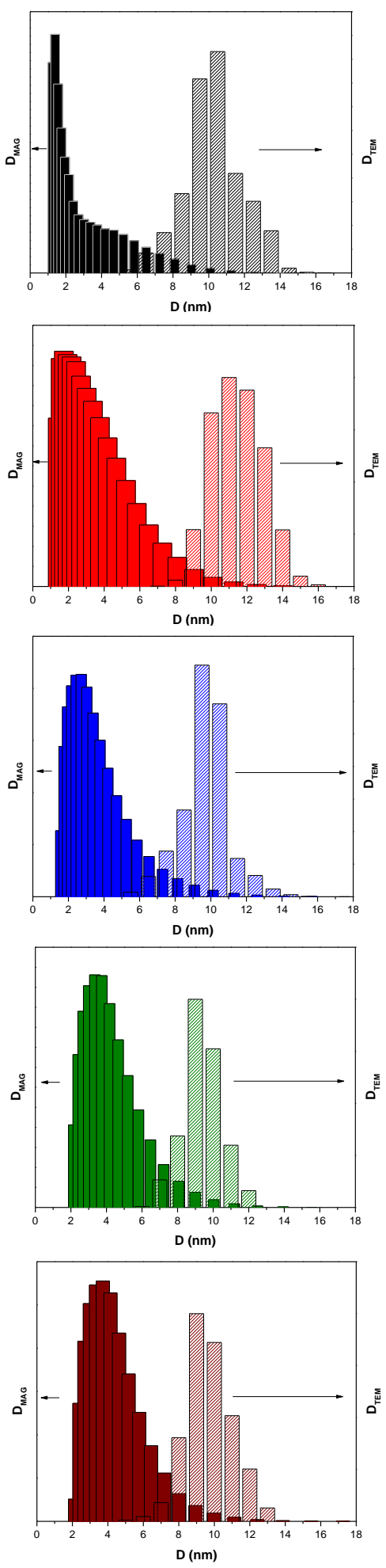

Figure S7 Comparison between number-weighted TEM and DCM size distributions obtained by MINORIM with the following conditions: a cut off of $1500000 \mathrm{~A} / \mathrm{m}$ for correcting the diamagnetic contribution; minimum and maximum dipole moments of $1 \cdot 10^{-22}$ and $1 \cdot 10^{-17} \mathrm{~A} \cdot \mathrm{m}^{2}$, respectively; 36 bins for 6 subsets; Langevin dipole domain mode. 

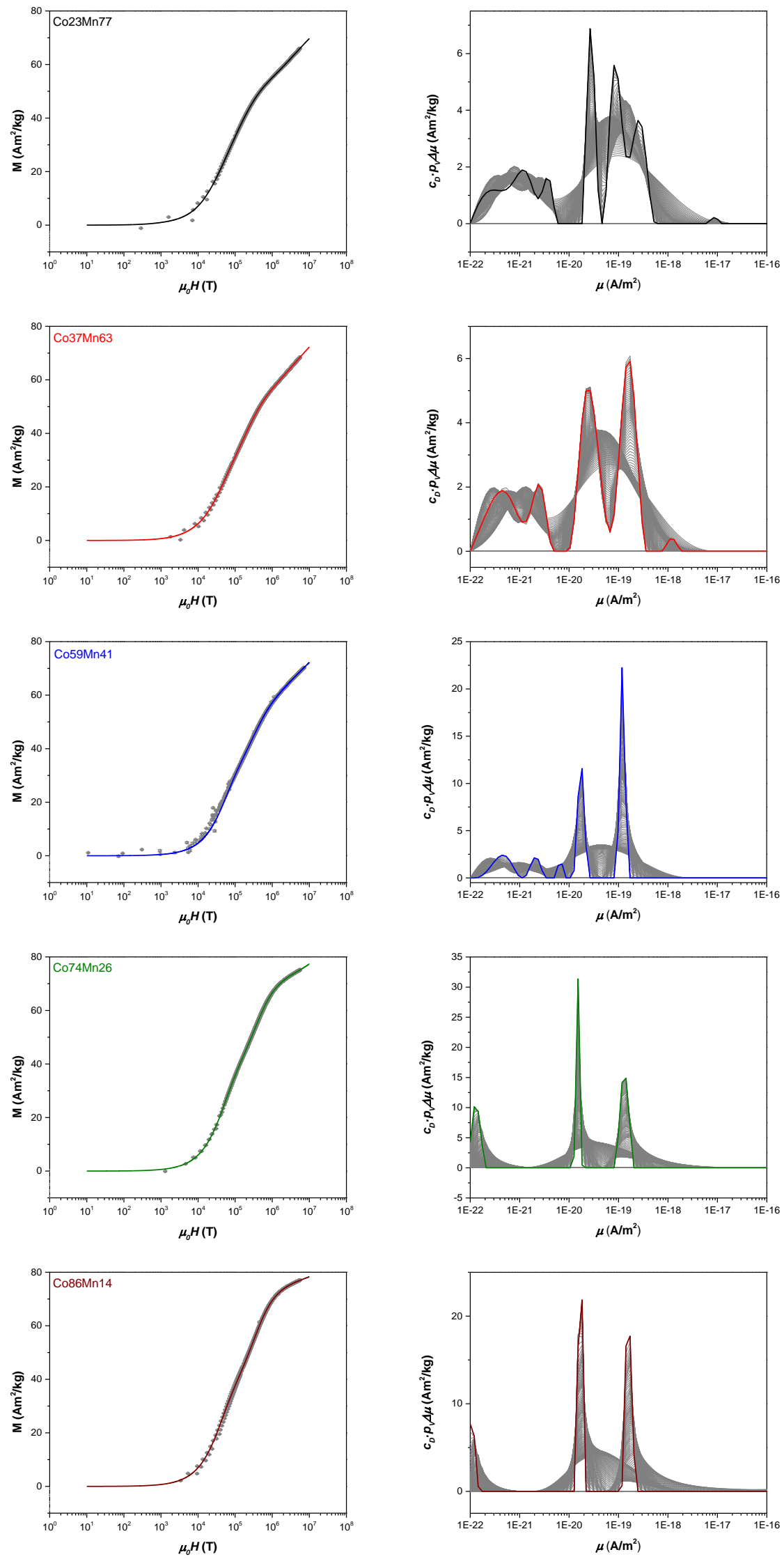

Figure S8 Field-dependent magnetization refinement of the $300 \mathrm{~K}$ magnetic field dependence of the magnetization for the Co-Mn mixed ferrite samples by using the numerical inversion reported in ref. ${ }^{2,3}$ (left), and resulting moment distributions (gray curves: 200 discrete moment distributions $\mathrm{c}_{\mathrm{D}} \cdot \mathrm{p}_{\mathrm{v}} \Delta \mu$; colored tick curve: moment distribution obtained for the highest evidence). 
Slight deviations between the fitting and the experimental curves are observed with both methods if a logarithmic scale for the magnetic field axis is adopted, as shown in Figure S5 and Figure S8. In particular, in the curve fitting by MINORIM (Figure S5), for the Mn-rich samples (Co23Mn77, Co37Mn63, Co59Mn41), a deviation is observed just for the first two points, which can be more affected by residual magnetic fields. More importantly, for both methods, in the case of the Co74Mn26 and Co86Mn14 samples, the experimental curves seem not to be characterised by a continuous ramp of the magnetisation as a function of the magnetic field, with the presence of a shoulder. In order to verify if this could be related to chemical composition inhomogeneities in these Co-Mn mixed ferrites, the same procedure was carried out on a sample of cobalt ferrite (a replica of the Co sample) and a 1:1 mechanical mixture of $\mathrm{CoFe}_{2} \mathrm{O}_{4}$ and $\mathrm{MnFe}_{2} \mathrm{O}_{4}$ nanoparticles of similar sizes (whose characterization is reported elsewhere ${ }^{4,5}$ ), as reported in Figure S9.
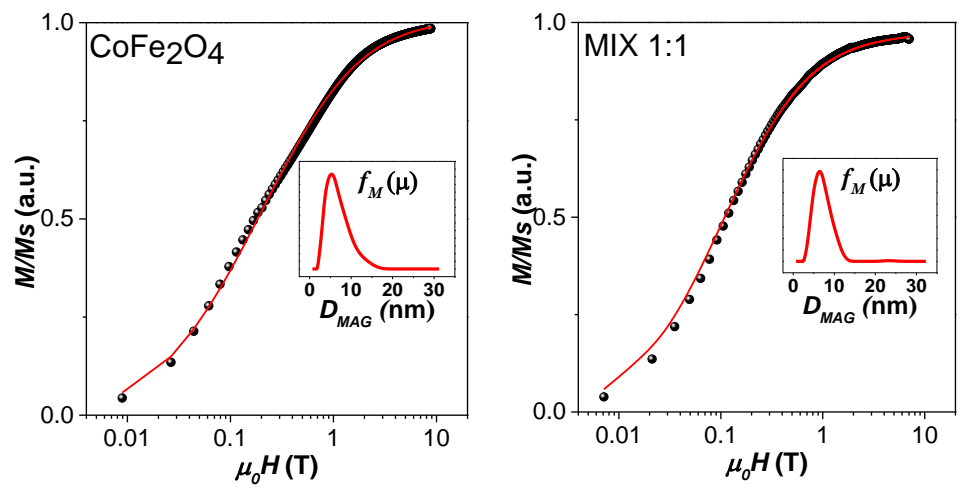

Figure S9 Field-dependent magnetization refinement of the $300 \mathrm{~K}$ magnetic field dependence of the magnetization for cobalt ferrite nanoparticles (left) and a 1:1 mechanical mixture of $\mathrm{CoFe}_{2} \mathrm{O}_{4}$ and $\mathrm{MnFe}_{2} \mathrm{O}_{4}$ nanoparticles of similar sizes by using MINORIM. ${ }^{1}$

The graphs show for the $\mathrm{CoFe}_{2} \mathrm{O}_{4}$ sample a similar behaviour to what observed for the Co74Mn26 and Co86Mn14 samples with a shoulder at about 0.1 in the field log scale, which is not visible for the mechanical mixture, meaning that this behaviour cannot be ascribed to the presence of separated ferrites with different composition, but it could be associated with the presence of $\mathrm{Co}^{2+}$ ions and its high single-ion anisotropy.

The deviation from the Langevin function may be due to more abundant nanoparticles in the blocked state in these samples, in agreement with an increase of the magnetic anisotropy and dipolar interactions. This is also confirmed by the $\mathrm{T}_{\text {diff }}$ values, which are above $300 \mathrm{~K}$ for the Co-rich samples (Table 2) and $\mathrm{CoFe}_{2} \mathrm{O}_{4}{ }^{5}$ 


\section{S5. DCD-IRM measurements}
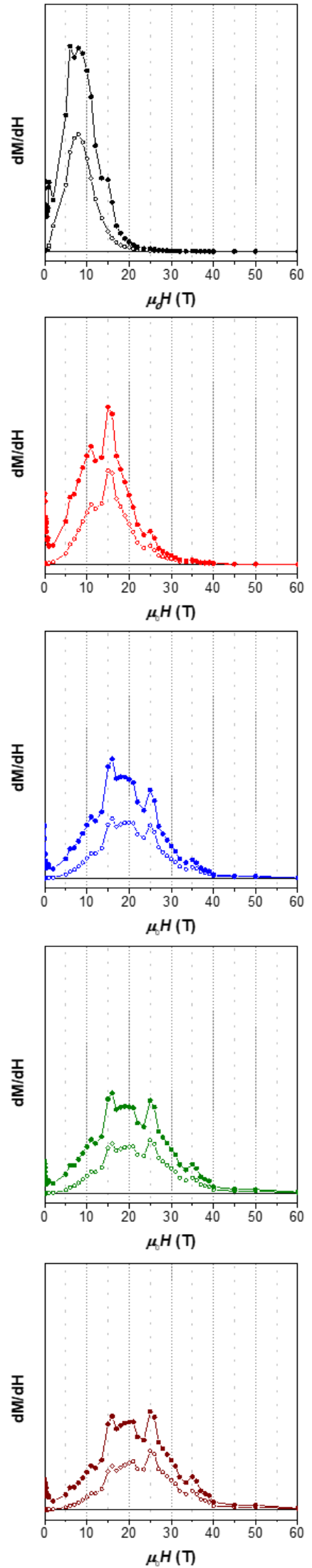

Figure S10 First derivative of the DCD (full circles) and IRM (empty circles) curves for the Co-Mn mixed ferrite samples. 


\section{S6. Hypothesis of cation distribution based on the Nèel model}

Table S1 Hypothesised cation distribution based on anisotropy constant and saturation magnetisation values for the CoMn mixed ferrite samples.

\begin{tabular}{|c|c|c|c|c|c|c|}
\hline Sample & $\begin{array}{c}\mathbf{M n}(\mathbf{I I})_{\text {theo }} \\
\left(\mathbf{T}_{\mathbf{d}}\right)\end{array}$ & $\begin{array}{c}\mathbf{C o}(\mathbf{I I})_{\text {theo }} \\
\left(\mathbf{T}_{\mathbf{d}}\right)\end{array}$ & $\begin{array}{c}\mathbf{F e}(\mathbf{I I I})_{\text {theo }} \\
\left(\mathbf{T}_{\mathbf{d}}\right)\end{array}$ & $\begin{array}{c}\mathbf{M n}(\mathbf{I I})_{\text {theo }} \\
\left(\mathbf{O}_{\mathbf{h}}\right)\end{array}$ & $\begin{array}{c}\mathbf{C o}(\mathbf{I I})_{\text {theo }} \\
\left(\mathbf{O}_{\mathbf{h}}\right)\end{array}$ & $\begin{array}{c}\mathbf{F e}(\mathbf{I I I})_{\text {theo }} \\
\left(\mathbf{O}_{\mathbf{h}}\right)\end{array}$ \\
\hline Co23Mn77 & 0.29 & 0 & 0.73 & 0.34 & 0.20 & 1.38 \\
\hline Co37Mn63 & 0.30 & 0 & 0.71 & 0.23 & 0.32 & 1.40 \\
\hline Co59Mn41 & 0.16 & 0 & 0.83 & 0.19 & 0.49 & 1.30 \\
\hline Co74Mn26 & 0 & 0.12 & 0.88 & 0.22 & 0.53 & 1.23 \\
\hline Co86Mn14 & 0 & 0.16 & 0.83 & 0.15 & 0.60 & 1.24 \\
\hline
\end{tabular}

Table S2 Comparison between the cation distribution obtained by ${ }^{57} \mathrm{Fe}$ Mössbauer spectroscopy (Möss) for the divalent cations (M(II)) and ferric ions and that obtained by the hypothesised one on the basis of anisotropy constant and saturation magnetisation values.

\begin{tabular}{|c|c|c|c|c|c|c|c|c|}
\hline Sample & $\begin{array}{c}\mathbf{M}(\mathbf{I I})_{\text {Möss }} \\
\left(\mathbf{T}_{\mathbf{d}}\right)\end{array}$ & $\begin{array}{c}\mathbf{M}(\mathbf{I I})_{\text {theo }} \\
\left(\mathbf{T}_{\mathbf{d}}\right)\end{array}$ & $\begin{array}{c}\mathbf{F e}(\mathbf{I I I})_{\mathbf{M o ̈ s s}} \\
\left(\mathbf{T}_{\mathbf{d}}\right)\end{array}$ & $\begin{array}{c}\mathbf{F e}(\mathbf{I I I})_{\text {theo }} \\
\left(\mathbf{T}_{\mathbf{d}}\right)\end{array}$ & $\begin{array}{c}\mathbf{M}(\mathbf{I I})_{\mathbf{M o ̈ s s}} \\
\left(\mathbf{O}_{\mathbf{h}}\right)\end{array}$ & $\begin{array}{c}\mathbf{M}(\mathbf{I I})_{\text {theo }} \\
\left(\mathbf{O}_{\mathbf{h}}\right)\end{array}$ & $\begin{array}{c}\mathbf{F e}(\mathbf{I I I})_{\mathbf{M o ̈ s s}} \\
\left(\mathbf{O}_{\mathbf{h}}\right)\end{array}$ & $\begin{array}{c}\mathbf{F e}(\mathbf{I I I})_{\text {theo }} \\
\left(\mathbf{O}_{\mathbf{h}}\right)\end{array}$ \\
\hline Co23Mn77 & 0.25 & 0.29 & 0.73 & 0.73 & 0.59 & 0.54 & 1.38 & 1.38 \\
\hline Co37Mn63 & 0.28 & 0.30 & 0.70 & 0.71 & 0.55 & 0.55 & 1.41 & 1.40 \\
\hline Co59Mn41 & 0.16 & 0.16 & 0.83 & 0.83 & 0.67 & 0.68 & 1.28 & 1.30 \\
\hline Co74Mn26 & 0.14 & 0.12 & 0.85 & 0.88 & 0.76 & 0.75 & 1.22 & 1.23 \\
\hline Co86Mn14 & 0.14 & 0.16 & 0.85 & 0.83 & 0.76 & 0.75 & 1.22 & 1.24 \\
\hline
\end{tabular}

Table S3 Hypothesised distribution for the metal cations (sum of divalent cations (M(II)) and ferric ions) in the tetrahedral and octahedral sites based on anisotropy constant and saturation magnetisation values for the Co-Mn mixed ferrite samples. V represents the vacancies obtained as difference between the ideal values of cation distribution ( 1 for tetrahedral and 2 for octahedral sites, respectively) and the hypothesised ones.

\begin{tabular}{|c|c|c|c|c|}
\hline Sample & $\mathbf{M}\left(\mathbf{T}_{\mathbf{d}}\right)$ & $\mathbf{M}\left(\mathbf{O}_{\mathbf{h}}\right)$ & $\mathbf{V}\left(\mathbf{T}_{\mathbf{d}}\right)$ & $\mathbf{V}\left(\mathbf{O}_{\mathbf{h}}\right)$ \\
\hline Co23Mn77 & 1.02 & 1.92 & - & 0.08 \\
\hline Co37Mn63 & 1.01 & 1.95 & - & 0.05 \\
\hline Co59Mn41 & 0.99 & 1.98 & 0 & 0 \\
\hline Co74Mn26 & 1.00 & 2.00 & 0 & 0 \\
\hline Co86Mn14 & 0.99 & 1.99 & 0 & 0 \\
\hline
\end{tabular}

Table S4 Comparison between theoretical and experimental contents per formula unit for Co, Mn, and Fe cations, anisotropy constant $(\mathrm{K})$, and saturation magnetisation $\left(\mathrm{M}_{\mathrm{s}}\right)$.

\begin{tabular}{|c|c|c|c|c|c|c|c|c|c|c|}
\hline Sample & Coexp & Cotheo & $\mathbf{M n}_{\exp }$ & $\mathbf{M n}_{\text {theo }}$ & $\mathbf{F e}_{\mathbf{e x p}}$ & $\mathbf{F e}_{\text {theo }}$ & $\begin{array}{c}\mathbf{K}_{\exp } \\
\left(\mathbf{1 0}_{\mathbf{5}} \mathbf{J} / \mathbf{m}^{\mathbf{3}}\right)\end{array}$ & $\begin{array}{c}\mathbf{K}_{\text {theo }} \\
\left(\mathbf{1 0}^{-22} \mathbf{J} / \mathbf{i o n}\right)\end{array}$ & $\begin{array}{c}\mathbf{M}_{\text {sexp }} \\
\left(\mathbf{A m}^{\mathbf{2}} / \mathbf{k g}\right)\end{array}$ & $\begin{array}{c}\mathbf{M}_{\text {stheo }} \\
\left(\mathbf{A m ^ { 2 }} / \mathbf{k g}\right)\end{array}$ \\
\hline Co23Mn77 & 0.19 & 0.20 & 0.65 & 0.63 & 2.11 & 2.11 & 3.74 & 1.7 & 98 & 100 \\
\hline Co37Mn63 & 0.31 & 0.32 & 0.52 & 0.53 & 2.11 & 2.11 & 6.37 & 2.7 & 98 & 99 \\
\hline Co59Mn41 & 0.49 & 0.49 & 0.34 & 0.35 & 2.11 & 2.13 & 9.00 & 4.2 & 95 & 96 \\
\hline Co74Mn26 & 0.66 & 0.65 & 0.23 & 0.22 & 2.07 & 2.11 & 9.99 & 4.4 & 98 & 99 \\
\hline Co86Mn14 & 0.77 & 0.76 & 0.13 & 0.15 & 2.07 & 2.07 & 10.9 & 5.0 & 99 & 97 \\
\hline
\end{tabular}




\section{References}

(1) Van Rijssel, J.; Kuipers, B. W. M.; Erné, B. H. Non-Regularized Inversion Method from Light Scattering Applied to Ferrofluid Magnetization Curves for Magnetic Size Distribution Analysis. $J$. Magn. Magn. Mater. 2014, 353, 110-115.

(2) Bender, P.; Balceris, C.; Ludwig, F.; Posth, O.; Bogart, L. K.; Szczerba, W.; Castro, A.; Nilsson, L.; Costo, R.; Gavilán, H.; et al. Distribution Functions of Magnetic Nanoparticles Determined by a Numerical Inversion Method. New J. Phys. 2017, 19 (7), 73012.

(3) Bender, P.; Bogart, L. K.; Posth, O.; Szczerba, W.; Rogers, S. E.; Castro, A.; Nilsson, L.; Zeng, L. J.; Sugunan, A.; Sommertune, J.; et al. Structural and Magnetic Properties of Multi-Core Nanoparticles Analysed Using a Generalised Numerical Inversion Method. Sci. Rep. 2017, 7 (April), 1-14.

(4) Sanna Angotzi, M.; Musinu, A.; Mameli, V.; Ardu, A.; Cara, C.; Niznansky, D.; Xin, H. L.; Cannas, C. Spinel Ferrite Core-Shell Nanostructures by a Versatile Solvothermal Seed-Mediated Growth Approach and Study of Their Nanointerfaces. ACS Nano 2017, 11 (8), 7889-7900.

(5) Sanna Angotzi, M.; Mameli, V.; Cara, C.; Musinu, A.; Sangregorio, C.; Niznansky, D.; Xin, H. L.; Vejpravova, J.; Cannas, C. Coupled Hard-Soft Spinel Ferrite-Based Core-Shell Nanoarchitectures: Magnetic Properties and Heating Abilities. Nanoscale Adv. 2020, 2 (8), 3191-3201. 